\title{
Vaccini Covid-19: disponibilità ed efficacia
}

\author{
RICCARDO DAVANZO \\ IRCCS Materno-Infantile "Burlo Garofolo", Trieste
}

Le grandi aspettative sul controllo della pandemia COVID-19 dipendono dalla campagna vaccinale in corso. II suo successo potrebbe però essere minacciato dalla lentezza della copertura vaccinale massimale e dall'insorgenza di varianti virali. I tempi per la vaccinazione in età pediatrica sono al momento prematuri e se ne discutono $i$ motivi. Il punto complessivo della situzione aggiornato al mese di Marzo.

A lla data del 6 marzo 2021 il Covid19 ha causato nel mondo oltre 115 milioni di infezioni e oltre 2,5 milioni di morti ${ }^{1}$. Per uscire dalla pandemia, grandi aspettative sono state da subito riposte sul vaccino anti-SARS-CoV-2. In data 23 aprile 2020, pochi mesi dopo il sequenziamento genetico di SARSCoV-2, l'OMS già contava 83 potenziali candidati per il vaccino anti-Covid ${ }^{1}$. Il presente articolo vuole fornire un sintetico aggiornamento e qualche risposta sui vaccini anti-COVID rispetto alla puntualizzazione di Marchetti e Guiducci $^{2}$ e agli interrogativi posti da Assael $^{3}$ sulle pagine di questa rivista rispettivamente a maggio e a dicembre 2020.

\section{VACCINI DISPONIBILI}

Un enorme sforzo organizzativo ed economico ha portato la ricerca scientifica a rendere concretamente disponibili nel mondo in meno di un anno una decina di vaccini anti-Covid-19, appartenenti a 4 distinte categorie a base rispettivamente di: 1) mRNA codificante per la proteina $\mathrm{S}$, protetto da nano-particelle lipidiche; 2) adenovirus reso privo della capacità di replicarsi, vettore del DNA codificante per la proteina $\mathrm{S}$; 3 ) sub-unità proteica $\mathrm{S}$ ricombinante del coronavirus; 4) coronavirus inattivato (Tabella I). Lo sviluppo di un vaccino con SARS-CoV-2 attenuato resta invece al momento in secondo piano.

COVID VACCINES: AVAILABILITY AND EFFICACY

(Medico e Bambino 2021;40(3):181-185 doi: 10.53126/MEB40181)

\section{Key words}

Covid-19, Vaccine, Viral variants, Paediatric population

\section{Summary}

The Covid-19 vaccination campaign represents a challenge for the health system. Eight Covid-19 vaccines belonging to 4 different categories of technology are currently available. More than the specific type of selected vaccine, major influencing factors for the success of the vaccination campaign are a quick coverage of the population, including the paediatric one, and the surveillance regarding the new viral variants.

\section{FATTORI INFLUENZANTI L'IMPATTO DELLA CAMPAGNA VACCINALE}

Il successo della campagna vaccinale è condizionato non tanto e non solo dalla scelta di uno specifico tipo di vaccino anti-Covid, quanto dalla capacità di giungere il più rapidamente possibile alla massima copertura vaccinale. Questa è influenzata a sua volta da fattori logistico-organizzativi come la disponibilità complessiva di vaccini (problema di grande attualità a febbraiomarzo 2021 in Italia e in Europa), le modalità di conservazione, distribuzione e somministrazione del prodotto, l'eventuale necessità di iniettare una seconda dose.

Di recente però si è aggiunto il fatto inquietante che un vaccino, pur diffusamente somministrato, possa funzionare meno per le varianti virali emergenti ${ }^{6}$, come suggerito da una ridotta efficacia complessiva in Sud Africa del vaccino
Johnson \& Johnson (57\% a fronte di un $72 \%$ negli USA) (Tabella I).

L'epidemiologo statunitense Michael Osterholm, consulente del Presidente Biden sul coronavirus, è giunto al punto di enfatizzare il rischio, quantomeno per gli USA, di un prossimo "uragano Covid", alimentato appunto dall'insorgere delle nuove mutazioni virali in combinazione con il ritardo dell'avanzamento della campagna vaccinale, con il rilassamento presso una popolazione stanca delle misure di prevenzione e con la documentata possibilità di reinfettarsi ${ }^{7}$. Per battere questo uragano sono quindi necessarie non solo una maggior rapidità nella somministrazione dei vaccini, ma anche una più stretta sorveglianza genomica sulle varianti e infine, ma non meno importante, l'adesione tempestiva, costante e diligente senza mai abbassare la guardia sulle misure di mitigazione di volta in volta suggerite dalle Autorità sanitarie. 


\begin{tabular}{|c|c|c|c|c|c|c|c|}
\hline \multicolumn{8}{|c|}{ INFORMAZIONI ESSENZIALI SUI VACCINI CONTRO SARS-COV-2 } \\
\hline Vaccino & Tipo & Antigene & Dosi & Distanza & Conservazione & $\begin{array}{l}\text { Efficacia contro } \\
\text { grave Covid-19 }\end{array}$ & Efficacia complessiva \\
\hline $\begin{array}{l}\text { mRNA-1 } 273 \\
\text { Moderna (USA) }\end{array}$ & mRNA & $\begin{array}{l}\text { Proteina spike } \\
\text { intera (S) }\end{array}$ & 2 & 4 sett. & $\begin{array}{l}-25 /-15^{\circ} \mathrm{C} ; \\
2-8{ }^{\circ} \mathrm{C} \text { per } 30 \mathrm{gg} ; \\
\text { temperatura } \\
\text { ambiente: } 12 \mathrm{~h}\end{array}$ & $\begin{array}{l}100 \% \text { dopo } 14 \mathrm{gg} \\
\text { dalla } 2^{\circ} \text { dose }\end{array}$ & $\begin{array}{l}92,1 \% \text { dopo } 1^{a} \text { dose; } \\
94,1 \% \text { dopo } 2^{a} \text { dose }\end{array}$ \\
\hline $\begin{array}{l}\text { BNT162b2 } \\
\text { Pfizer-BionNTech } \\
\text { (USA) }\end{array}$ & mRNA & $\begin{array}{l}\text { Proteina spike } \\
\text { intera (S) }\end{array}$ & 2 & 3 sett. & $\begin{array}{l}-80 /-60^{\circ} \mathrm{C} ; \\
2-8^{\circ} \mathrm{C} \text { per } 5 \mathrm{gg} ; \\
\text { temperatura } \\
\text { ambiente: } 2 \mathrm{~h}\end{array}$ & $\begin{array}{l}88,9 \% \text { dopo } \\
1^{a} \text { dose }\end{array}$ & $\begin{array}{l}52 \% \text { dopo } 1^{a} \text { dose; } \\
94,6 \% \text { dopo la } 2^{a} \text { dose }\end{array}$ \\
\hline $\begin{array}{l}\text { Ad26.CoV2.S } \\
\text { Johnson \& Johnson } \\
\text { (USA) }\end{array}$ & $\begin{array}{l}\text { Vettore } \\
\text { virale }\end{array}$ & $\begin{array}{l}\text { Adenovirus } 26 \\
\text { incapace di } \\
\text { replicarsi } \\
\text { codificante } \\
\text { la proteina S }\end{array}$ & 1 & - & $\begin{array}{l}-20^{\circ} \mathrm{C} \\
2-8^{\circ} \mathrm{C} \text { per } 3 \text { mesi }\end{array}$ & $\begin{array}{l}85 \% \text { dopo } 4 \text { sett.; } \\
100 \% \text { dopo } \\
49 \text { giorni }\end{array}$ & $\begin{array}{l}72 \% \text { in USA } \\
66 \% \text { in Sudamerica } \\
57 \% \text { in Sud Africa } \\
\text { (a } 28 \mathrm{gg} \text { ) }\end{array}$ \\
\hline $\begin{array}{l}\text { ChAdOxl } \\
\text { (AZS1222) } \\
\text { AstraZeneca } \\
\text { (Regno Unito) }\end{array}$ & $\begin{array}{l}\text { Vettore } \\
\text { virale }\end{array}$ & $\begin{array}{l}\text { Adenovirus di } \\
\text { scimpanzé } \\
\text { incapace di } \\
\text { replicarsi } \\
\text { codificante la } \\
\text { proteina S } \\
\end{array}$ & 2 & $4-12$ sett. & $2-8^{\circ} \mathrm{C}$ per 6 mesi & $\begin{array}{l}100 \% \text { dopo } \\
\text { la } 1^{a} \text { dose }\end{array}$ & $\begin{array}{l}64,1 \% \text { dopo } 1 \text { sett. } \\
70,4 \% \text { dopo } 2 \text { sett. dalla } \\
2^{\mathrm{a}} \text { dose }\end{array}$ \\
\hline $\begin{array}{l}\text { Gam-COVID-Vac } \\
\text { (Sputnik) } \\
\text { (Russia) }\end{array}$ & $\begin{array}{l}\text { Vettore } \\
\text { virale }\end{array}$ & $\begin{array}{l}\text { Adenovirus } \\
\text { incapace di } \\
\text { replicarsi } \\
\text { codificante la } \\
\text { glicoproteina S }\end{array}$ & $\begin{array}{l}2 \text { (prima } \\
\text { AD26, } \\
\text { seconda } \\
\text { AD5) }\end{array}$ & 3 sett. & $\begin{array}{l}-18{ }^{\circ} \mathrm{C} \\
2-8^{\circ} \mathrm{C} \text { per } 6 \text { mesi }\end{array}$ & $\begin{array}{l}100 \%, 3 \text { sett. } \\
\text { dopo la } 1^{\circ} \text { dose }\end{array}$ & $\begin{array}{l}87,6 \%, 1 \text { sett. } \\
\text { dopo la } 1^{a} \text { dose } \\
91,1 \% 7 \mathrm{gg} \text { dopo } \\
\text { la } 2^{\mathrm{a}} \text { dose }\end{array}$ \\
\hline $\begin{array}{l}\text { NVX-CoV2373 } \\
\text { Novavax (USA) }\end{array}$ & $\begin{array}{l}\text { Subunità } \\
\text { proteica }\end{array}$ & $\begin{array}{l}\text { Proteina S intera, } \\
\text { ricombinante }\end{array}$ & 2 & - & $2-8^{\circ} \mathrm{C}$ per 6 mesi & Sconosciuta & $\begin{array}{l}89,3 \% \text { in UK dopo } 2 \text { dosi } \\
60 \% \text { in Sud Africa }\end{array}$ \\
\hline $\begin{array}{l}\text { CoronaVac } \\
\text { Sinovac Biotech } \\
\text { (Cina) }\end{array}$ & \begin{tabular}{l|} 
Virus \\
inattivato
\end{tabular} & $\begin{array}{l}\text { Ceppo CNO2 } \\
\text { inattivato }\end{array}$ & 2 & 2 sett. & $2-8^{\circ} \mathrm{C}$ & Sconosciuta & $\begin{array}{l}\text { Dati non ancora pubblicati } \\
\text { Riportata efficacia } \\
\text { variabile dopo } 2 \text { sett.: } \\
65-91 \%\end{array}$ \\
\hline $\begin{array}{l}\text { BBIBP-CoV } \\
\text { Sinopharm 1/1 } \\
\text { (Cina) }\end{array}$ & $\begin{array}{l}\text { Virus } \\
\text { inattivato }\end{array}$ & $\begin{array}{l}\text { Ceppo HBO2 } \\
\text { inattivato }\end{array}$ & 2 & 3 sett. & $2-8^{\circ} \mathrm{C}$ & Sconosciuta & $\begin{array}{l}\text { Dati non ancora pubblicati } \\
\text { Riportata efficacia } \\
\text { variabile: } 79-86 \%\end{array}$ \\
\hline
\end{tabular}

Nota: I vaccini italiani Covid-eVax a DNA della Takis e GRAd-CoV-2 con adenovirus di gorilla della ReiThera a inizio marzo 2021 non hanno ancora completato le 3 fasi di studi clinici richieste per l'autorizzazione d'impiego.

Tabella I. Da voci bibliografiche 4 e 5, modificata.

\section{REINFEZIONI}

La durata della protezione ottenuta dopo infezione naturale da SARS-CoV2 è stata messa in discussione dal fatto che sono possibili reinfezioni da SARS$\mathrm{CoV}-2$, anche se solitamente di minor gravità ${ }^{8}$. A tale proposito vanno debitamente considerate alcune evidenze scientifiche:

1. nessuno degli 11.000 operatori sanitari inglesi ammalatisi di Covid-19 durante la prima ondata pandemica fra marzo e aprile 2020 ha presentato reinfezioni sintomatiche durante la seconda ondata di ottobre-novembre
2020, suggerendo che l'immunità possa durare almeno 6 mesi ;

2. la Public Health England indica che, su 6614 operatori sanitari Covid-19, solo 44 hanno avuto una possibile/ probabile (quindi non certa!) infezione, suggerendo una frequenza del fenomeno inferiore a $1 \%^{10}$;

3. a livello mondiale a marzo 2021 sono riportati complessivamente in letteratura meno di 100 casi di reinfezione accertata da Covid-19.

In sintesi, i dati sulla reale frequenza delle reinfezioni sono limitati, anche perché la disponibilità di se- quenziamento genomico del SARS$\mathrm{CoV}-2$, unico modo per discriminare fra reinfezione o riattivazione, è ancora limitata ${ }^{8}$.

\section{VARIANTI DEL CORONAVIRUS}

SARS-CoV-2 è un virus a RNA che costantemente evolve mediante comparsa di mutazioni genetiche. Solo una minoranza fra le molte migliaia di mutazioni che si manifestano è capace di fornire al virus un vantaggio selettivo, facilitando la diffusione di nuove varianti virali (Tabella II). Queste sono 


\begin{tabular}{|c|c|c|c|}
\hline \multicolumn{4}{|c|}{ CARATTERISTICHE ESSENZIALI DELLE VARIANTI VIRALI CHE DESTANO PREOCCUPAZIONE (VOC) } \\
\hline Linea secondo PANGOLIN* & B.1.1.7 & B.1.351 & B.1.1.28.1 \\
\hline Primo Paese in cui è stato isolato & Regno Unito & Sud Africa & Brasile/Giappone \\
\hline Prima comparsa & Settembre 2020 & Agosto 2020 & Dicembre 2020 \\
\hline Mutazioni chiave di spike & $\begin{array}{l}\text { H69/V70 deletion; } \\
\text { Y144 deletion; } \\
\text { N501Y; A570D; } \\
\text { D614G; e P681H }\end{array}$ & $\begin{array}{l}\text { L242/A243/L244 deletion; } \\
\text { N501Y; D614G; E484K; and K417N }\end{array}$ & $\begin{array}{l}\text { N501Y; D614G; E484K; } \\
\text { e K417N }\end{array}$ \\
\hline Mutazioni chiave in comune & \multicolumn{3}{|c|}{ S106/G107/F108 deletion in Non-Structural Protein 6 (NSP6) } \\
\hline Trasmissibilità & $\begin{array}{l}\text { Aumentata del } 36-75 \% \text {; } \\
\text { aumento del tasso secondario } \\
\text { di attacco }(10-13 \%)\end{array}$ & Aumentato di 1,5 volte & Suggerito come aumentato \\
\hline Gravità & $\begin{array}{l}\text { Ė possibile sia aumentata, } \\
\text { come pure la mortalità }\end{array}$ & Non viene riportata alcuna variazione & $\begin{array}{l}\text { Al momento non viene } \\
\text { riportata alcuna variazione }\end{array}$ \\
\hline Capacità di neutralizzazione & $\begin{array}{l}\text { Leggermente ridotta, } \\
\text { ma sufficiente a conferire } \\
\text { protezione }\end{array}$ & $\begin{array}{l}\text { Ridotta; suggerito aumentato rischio } \\
\text { di reinfezione }\end{array}$ & $\begin{array}{l}\text { Potenziale calo; riportati casi } \\
\text { di reinfezione }\end{array}$ \\
\hline $\begin{array}{l}\text { Impatto potenziale } \\
\text { sulla vaccinazione }\end{array}$ & $\begin{array}{l}\text { Nessun significativo impatto } \\
\text { sui vaccini Moderna, } \\
\text { Pfizer-BioNTech, } \\
\text { e Oxford-AstraZeneca }\end{array}$ & $\begin{array}{l}\text { Moderna e Pfizer-BioNTech: riduzione } \\
\text { attività neutralizzante, ma l'impatto } \\
\text { sulla malattia non è noto. } \\
\text { Novavax e Johnson \& Johnson: } \\
\text { efficacia dei vaccini sulle forme moderate- } \\
\text { gravi inferiore in Sud Africa rispetto ai } \\
\text { Paesi senza varianti. In attesa dei risultati } \\
\text { sierologici sull'attività neutralizzante. } \\
\text { Oxford/AstraZeneca: efficacia limitata } \\
\text { del vaccino contro forme lievi-moderate } \\
\text { di Covid-19 ed efficacia da determinarsi } \\
\text { per le forme gravi. Capacità neutralizzante } \\
\text { sierologica sostanzialmente ridotta rispetto } \\
\text { ai ceppi originali (studio su piccoli } \\
\text { campioni) }\end{array}$ & Studi in corso \\
\hline $\begin{array}{l}\text { Paesi in cui sono stati segnalati casi } \\
\text { (di cui riportati nell'ultima settimana) }\end{array}$ & $107(7)$ & $51(5)$ & $29(8)$ \\
\hline
\end{tabular}

Tabella II. Aggiornamento al 23 febbraio 2021 (da voci bibliografiche 11 e 12, modificata).

secondo l'OMS motivo di preoccupazione (Variants of Concerns o VoC) quando a confronto del coronavirus di riferimento si siano riscontrati, non solo dei cambiamenti genotipici ma anche fenotipici relativi a epidemiologia, trasmissibilità, antigenicità, virulenza, presentazione clinica o cambiamenti che possano comunque influenzare la diagnostica di laboratorio, l'efficacia della vaccinazione o della terapia ${ }^{11,12}$.

La maggior parte dei batteri e di virus come l'HIV e l'HCV evolvono lentamente. Al contrario alcuni altri virus, come quello dell'Influenza A, e analogamente SARS-CoV-2, presentano un elevato turnover delle linee virali circo- lanti. Per il virus influenzale di tipo A, per esempio, la nuova diversità genetica emergente ogni anno prevale su quella dell'anno precedente.

Secondo una survey pubblicata sul numero di febbraio di Nature e che ha raccolto l'opinione di 100 studiosi fra immunologi, infettivologi e virologi, anche la pandemia Covid-19 è destinata, come l'influenza, a diventare negli anni un'endemia con andamento stagionale $^{13}$, con infezione meno grave (clinicamente simile a quella dell'età pediatrica) e con possibilità di venir eliminata in alcune aree del globo, che diventerebbero così Covid-free ${ }^{14}$.

La questione principale è se la gran- de variabilità genomica possa realmente modificare la risposta immune e di fatto pregiudicare l'efficacia dei vaccini Covid-19 recentemente sviluppati. I vaccini vivi attenuati come quello MMR o Sabin inducono risposte policlonali dirette a un certo numero di proteine virali. Si tratta di una risposta multipla umorale e cellulare, che non consente ai ceppi virali di sfuggire. Un'eccezione è costituita dal virus influenzale nel quale il drift antigenico (fenomeno della deriva per l'accumulo nel tempo di mutazioni che riguardano l'emoagglutinina e la neuraminidasi) e lo shift antigenico (la ricombinazione di queste proteine) fa sì che il vaccino 
non determini più l'effetto neutralizzante sul nuovo ceppo. Queste conoscenze sul virus influenzale suggeriscono che l'evoluzione di SARS-CoV-2 possa rendere meno efficace il vaccino, quando mutazioni riguardino proprio i due settori dello spike contro i quali è diretta la risposta anticorpale naturale o indotta dal vaccino: l'N-Terminal Domain (NTD) e il Receptor-Binding Domain (RBD). Quando un virus cresce sotto la pressione selettiva di un singolo anticorpo monoclonale che ha come bersaglio una sola epitope (determinante antigenico) dello spike originale di Wuhan-Hu-1, mutazioni che riguardano proprio questa epitope potrebbero però portare a riduzione o perdita dell'effetto neutralizzante dell'anticor$\mathrm{po}^{15}$.

Un elemento rassicurante è che la robusta risposta dei linfociti $\mathrm{T}$ ai vaccini contro SARS-CoV-2 è per natura policlonale con capacità di bersagliare porzioni differenti di spike. Fino a che punto questa risposta dell'immunità cellulare riesca a compensare quella umorale è attualmente oggetto di studio, anche se un preprint di Tarke e coll. del La Jolla Institute of Immuno$\log y$ in California comunica un dato incoraggiante: la risposta immunitaria cellulare $\mathrm{CD} 4+$ e CD8+ da parte dei sieri di convalescenti e degli immunizzati con i vaccini Moderna e Pfeizer/BioNTech risulta adeguata a neutralizzare quelle che al momento sono le 3 principali VoC: B.1.1.7, B.1.351 e P1 ${ }^{16}$.

Resta il fatto che qualora l'efficacia dell'immunizzazione risultasse vanificata da nuove future varianti virali, sarebbe comunque possibile riformulare periodicamente i vaccini per renderli "aggiornati”. I produttori Pfizer e AstraZeneca lo hanno già pianificato, mentre Moderna sta avviando un trial su un vaccino modificato presumibilmente più efficace sulla variante B. $1.351^{17}$.

\section{FASCE DI POPOLAZIONE NON TESTATE PER LA VACCINAZIONE}

Un altro elemento che potrebbe condizionare negativamente l'impatto della vaccinazione di massa sulla pan- demia è quello della possibile persistente circolazione di SARS-CoV-2 all'interno dei gruppi di non vaccinati. $\mathrm{Si}$ stima che sulla base degli attuali criteri di vaccinazione, questi potrebbero ammontare in Italia a oltre 10 milioni di persone, includendo i no-vax e gli individui esclusi perché in età pediatrica 0 in gravidanza. Come è noto il rapido processo di autorizzazione dei vaccini anti-Covid in uno stato di emergenza da parte dell'Autorità regolatoria non ha finora consentito di avere informazioni su sicurezza ed efficacia su alcuni particolari gruppi di popolazione.

La vaccinazione anti-Covid in allattamento, inizialmente controindicata, ha ora raccolto un ampio consenso internazionale $\mathrm{e}^{18,19} \mathrm{e}$ nazionale in base al criterio di sicurezza fornito dalla plausibilità biologica ${ }^{20}$. Lo stesso non vale per la vaccinazione anti-Covid in gravidanza, che al momento viene suggerita da OMS, CDC e ISS per le sole donne a elevato rischio di esposizione, come operatrici sanitarie o caregiver $^{21}$.

\section{VACCINARE LA POPOLAZIONE PEDIATRICA}

L'inclusione della popolazione sotto i 16-18 anni non è prioritaria nella fase iniziale di distribuzione dei vaccini anti-Covid. Infatti il Covid-19 in questa fascia di età è solitamente lieve o moderato, tranne che nei soggetti gravemente neuro-disabili ${ }^{22}$.

La vaccinazione di bambini e adolescenti consentirà di prevenire i rari casi di Covid-19 pediatrico grave (comprese le forme post-infettive come la sindrome infiammatoria multiorgano - MIS-C), faciliterà la ripresa della socializzazione scolastica, ludica e sportiva di bambini e adolescenti, proteggerà in maniera indiretta la popolazione non pediatrica per effetto della riduzione della circolazione del coronavirus, costruendo di fatto un'immunità di gregge $\mathrm{e}^{23}$.

Secondo Anthony Fauci, direttore del National Institute of Allergy and Infectious Diseases (USA), per avere informazioni su sicurezza ed efficacia dei vaccini anti-Covid in età pediatrica bisognerà aspettare almeno fino a

\section{MESSACGI CHIAVE}

$\square$ Il successo della campagna vaccinale Covid-19 risulta condizionata in maniera essenziale dalla rapidità di ottenere un'adeguata copertura della popolazione.

$\square$ Le reinfezioni da SARS-CoV-2 sono possibili, ma rappresentano al momento un fenomeno di scarso impatto epidemiologico.

$\square$ Le varianti virali (inglese, sudafricana e brasiliana) di SARS-CoV-2 possono influenzare l'efficacia della vaccinazione anti-Covid-19. Gli studi sono in corso, le evidenze non ancora robuste in termini di precise informazioni.

$\square$ Le case produttrici dei vaccini sembrano essere pronte per riformulare eventualmente $\mathrm{i}$ vaccini in base alle nuove varianti.

$\square$ Gli individui in età pediatrica $1<18$ anni) in prospettiva dovranno essere presumibilmente vaccinati, ma questo, da alcune stime, sembra che non sarà possibile prima del mese di gennaio 2022.

settembre 2021 quando saranno disponibili i risultati degli studi clinici su fasce di età progressivamente decrescenti: 16-12 anni, 12-9 anni e 9-6 anni $^{24}$. Lo stesso Fauci ritiene che la popolazione in età pediatrica potrà essere vaccinata a partire dall'inizio $2022^{25}$.

Proteggere l'età pediatrica dal Covid-19 è un obbligo etico e una necessità pratica, ma sarà necessario rassicurare preliminarmente i genitori sulla sicurezza del vaccino e far capire loro che questa scelta è saggia, preparandosi però allo stesso tempo a contrastare mediaticamente le prevedibili campagne di disinformazione ${ }^{26}$.

\section{Indirizzo per corrispondenza:}

Riccardo Davanzo

riccardo.davanzo@gmail.com 


\section{Bibliografia}

1. World Health Organization. Coronavirus Disease (Covid-19). Dashboard. https://covid19.who.int.

2. Marchetti F, Guiducci C. Vaccino e Covid19: cosa sappiamo. Medico e Bambino 2020;39 (5):297-9.

3. Assael BM. La vaccinazione anti-Covid... nello spazio e nel tempo. Medico e Bambino 2020;39(10):619-20.

4. Creech C, Walker SC, Samuels RJ. SARSCoV-2 vaccines. JAMA 2021 Feb 26, 2021 . doi: 10.1001/jama.2021. 3199. [Epub ahead of print]

5. Cennimo DJ. Covid-19 vaccines. Medscape. Updated: Feb 27, 2021.

6. Moore JP, Offit PA. SARS-CoV-2 vaccines and the growing threat of viral variants. JAMA 2021;325(9):821-2. doi: 10.1001/jama.2021. 1114 .

7. Kamisar B. Top epidemiologist says Biden administration needs to focus on first vaccine doses. "The hurricane is coming" Michael Olsterholm said. "We have to call an audible". NBC News. Jan 31, 2021.

8. Stokel-Walker C. What we know about Covid-19 reinfection so far. BMJ 2021;372:n99. doi: 10.1136/bmj.n99.

9. Hanrath AT, Payne BAI, Duncan CJA. Prior SARS-CoV-2 infection is associated with protection against symptomatic reinfection. J Infect 2020;S0163-4453(20)30781-7. doi: 10.1016/ j.jinf.2020.12.023.

10. Wilkinson E. Covid-19 reinfection "rare" says NHS study but some may still pass the virus on. Pulse 2021.
11. World Health Organization. Covid-19 weekly epidemiological update. Data as received by WHO from National Authorities, as of 21 February 2021, 10 am CET.

12. World Health Organization. Covid-19 weekly epidemiological update. Special edition: Proposed working definitions of SARSCoV-2 variants of interest and variants of concern. 25 February 2021.

13. Phillips N. The coronavirus is here to stay - here's what that means. Nature 2021;590:3824. doi:10.1038/d41586-021-00396-2.

14. Torjesen I. Covid-19 will become endemic but with decreased potency over time, scientists believe. BMJ 2021;372:n494. doi: 10.1136/bmj.n494 (Published 18 February 2021).

15. Williams TC, Burgers WA. SARS-CoV-2 evolution and vaccines: cause for concern? Lancet Respir Med 2021:S2213-2600(21) 00075-8. doi: 10.1016/S2213-2600(21)00075-8 [Epub ahead of print].

16. Tarke A, Sidney J, Methot N, et al. Negligible impact of SARS-CoV-2 variants on CD4+ and CD8+ T cell reactivity in Covid-19 exposed donors and vaccinees. bioRxiv posted 2021, March 1. doi: $10.1101 / 2021.02 .27 .433180$. 17. Mahase E. Covid-19: where are we on vaccines and variants? BMJ 2021;372:n597. doi: 10.1136/bmj.n597.

18. Chervenak FA, McCullough LB, Bornstein E, et al. Professionally responsible coronavirus disease 2019 vaccination counseling of obstetrical and gynecologic patients. Am J Obstet Gynecol 2021 Feb 1:S0002-9378(21)00082-X. doi: 10. 1016/j.ajog.2021.01.027 [Epub ahead of print]. 19. Merewood A, Bode L, Davanzo R, Perez-
Escamilla R. Breastfeed or be vaccinated - An unreasonable default recommendation. Lancet 2021;397(10274):578. doi: 10.1016/S01406736(21) 00197-5

20. Davanzo R, Agosti M, Cetin I, et al. Breastfeeding and Covid-19 vaccination: position statement of the Italian scientific Societies. Ital J Pediatr 2021;47(1):45. doi: 10.1186/s13052021-00998-6.

21. Istituto Superiore di Sanità. Indicazioni $a d$ interim su "Vaccinazione contro il Covid-19 in gravidanza e allattamento" - 09.01.2021. Italian Obstetric Surveillance System (ItOSS), aggiornamento del 31 gennaio 2021.

22. Shekerdemian LS, Mahmood NR, Wolfe KK et al. Characteristics and outcomes of children with coronavirus disease 2019 (Covid-19) infection admitted to US and Canadian pediatric intensive care Units. JAMA Pediatr 2020;174:868-73. doi:10.1001/jamapediatrics. 2020.1948 .

23. Wong BLH, Ramsay ME, Ladhani SN. Should children be vaccinated against Covid19 now? Arch Dis Child 2021 Jan 5:archdischild-2020-321225. doi: 10.1136/archdischild2020-321225 [Epub ahead of print].

24 . Chen C. Vaccines for kids as young as first graders could be authorized by September. ProPublica. Investigation Journalism in the Public Interest. 2021, Feb 11.

25. Crist C. Medscape News \& Perspectiv. Fauci: Children likely to receive Covid-19 vaccines in early 2022. March 1, 2021.

26. Klass P, Ratner AJ. Vaccinating children against Covid-19 - The lessons of measles. N Engl J Med 2021;384:589-91. doi: 10.1056/ NEJMp2034765. 